\title{
Aspectos bioecológicos de la ostra de agua dulce Acostaea rivoli (Mollusca, Etheriidae) en el río Opia, Tolima, Colombia
}

\author{
Nohora Efigenia Gutiérrez-Hoyos ${ }^{1, *}$, Edwin Orlando Lopez ${ }^{1}$, Gladys Reinoso-Florez ${ }^{1}$, \\ Jesús Manuel Vásquez-Ramos ${ }^{2}$, Francisco Villa-Navarro ${ }^{1}$

\begin{abstract}
${ }^{1}$ Grupo de Investigación en Zoología, Facultad de Ciencias, Universidad del Tolima, Ibagué, Colombia ${ }^{2}$ Universidad de Los Llanos, Grupo de Investigación Evaluación, Manejo y Conservación de Recursos Hidrobiológicos y Pesqueros (GHIPES), Facultad de Ciencias Básicas e Ingeniería, Departamento de Biología y Química, Villavicencio, Meta, Colombia
\end{abstract}

\section{Resumen}

La ostra de agua dulce Acostaea rivoli es una especie endémica de Colombia, que desempeña un papel ecológico relevante en el ecosistema acuático. El presente estudio se realizó en la cuenca del río Opia departamento del Tolima en los meses de septiembre y octubre de 2009, donde se establecieron 18 estaciones de muestreo, en cada una de las estaciones fueron empleados cuadrantes de $1 \mathrm{~m}^{2}$ distribuidos en diez transectos longitudinales. Adicionalmente, fue evaluada la distribución vertical entre 1 y $3 \mathrm{~m}$ de profundidad. Paralelamente se evaluaron variables fisicoquímicas in situ y se tomaron muestras de agua para la determinación de 12 variables fisicoquímicas. Para evaluar la relación entre la especie y las variables fisicoquímicas se realizó un análisis de componentes principales y una correlación de Spearman. Fueron observados 303 organismos, donde la mayor densidad se presentó en la estación Lorencito con 6,67 ind $/ \mathrm{m}^{2}$ y la menor densidad en las estaciones puente Doima, molino federal y quebrada Opia en donde no se registró ningún individuo. Con relación al perfil vertical la mayor densidad se presentó en el primer metro con 29,62 ind $/ \mathrm{m}^{2}$ y la menor en el tercer metro con 4,50 ind $/ \mathrm{m}^{2}$. Las variables fisicoquímicas que influyeron de forma significativa en la distribución y densidad de la población fueron la alcalinidad $(\mathrm{r}=0,698 ; \mathrm{p}=0,001)$, dureza $(\mathrm{r}=0,650 ; \mathrm{p}=0,004), \mathrm{pH}(\mathrm{r}=0,714 ; \mathrm{p}=0,001)$, cloruros $(\mathrm{r}=-0,530 ; \mathrm{p}=0,024)$, fosfatos $(\mathrm{r}=-0,603 ; \mathrm{p}=0,008)$, nitratos $(r=-0,490 ; p=0,039)$ y coliformes totales $(r=-0,540 ; p=0,021)$. Estas variables podrían estar relacionados con los procesos de mineralización de la cuenca, que junto con un alto porcentaje de calcio, una buena oxigenación del agua y un sustrato adecuado estarían relacionados con la presencia de esta especie en la zona de estudio. (C) 2017. Acad. Colomb. Cienc. Ex. Fis. Nat.

Palabras clave: Endémica; Ostra; Tolima; Variables fisicoquímicas.

Bioecological aspects of the freshwater oyster Acostaea rivoli (Mollusca, Etheriidae) in the Opia river, Tolima, Colombia

\begin{abstract}
The freshwater oyster (Acostaea rivoli) is an endemic Colombian species that plays an important ecological role in the aquatic ecosystem. The present study was performed in the Opia river basin, in the Tolima department. 18 sampling stations were evaluated during September and October of 2009. In each location quadrants of $1 \mathrm{~m}^{2}$ were distributed in ten longitudinal transects. Additionally, the vertical distribution was evaluated between 1 and $3 \mathrm{~m}$ deep. Parallelly, water samples were taken to analyzed 12 physicochemical variables, some of them were evaluated in situ and the others in the laboratory. In order to identify the relationships between the population and the physicochemical variables a principal component analysis and a Spearman correlation were performed. A total of 303 individuals were observed, the greatest density was recorded in the sampling site Lorencito with 6.67 ind/ $\mathrm{m}^{2}$ and the lowest $\left(0 \mathrm{ind} / \mathrm{m}^{2}\right.$ ) was recorded in the sites Puente Doima, Molino el Federal and Quebrada Opia. As regards to the vertical profile, the highest density was registered in the first meter with $29.62 \mathrm{ind} / \mathrm{m}^{2}$ and the lowest in the third meter with just $4.50 \mathrm{ind} / \mathrm{m}^{2}$. In relation to the water parameters,variables such as: alkalinity $(\mathrm{r}=0.698$; $\mathrm{p}=0.001)$, hardness $(\mathrm{r}=0.650 ; \mathrm{p}=0.004), \mathrm{pH}(\mathrm{r}=0.714 ; \mathrm{p}=0.001)$, chlorides $(\mathrm{r}=-0.530 ; \mathrm{p}=0.024)$, phosphates $(\mathrm{r}=$ $-0.603 ; \mathrm{p}=0.008)$, nitrates $(\mathrm{r}=-0.490 ; \mathrm{p}=0.039)$ and total coliforms $(\mathrm{r}=-0.540 ; \mathrm{p}=0.021)$ were significantly correlated with population's abundance and distribution. These variables could be related to mineralization processes in the basin, that combined with high values of calcium, high levels of water oxygen and proper habitats would allow this specie to inhabit the study area. (c) 2017. Acad. Colomb. Cienc. Ex. Fis. Nat.
\end{abstract}

Key words: Endemic; Oyster; Tolima; Physicochemical variable.

\footnotetext{
*Correspondencia:
}

Nohora E. Gutiérrez Hoyos, nohoragutierrezhoyos@outlook.com; Recibido: 09 de mayo de 2016; Aceptado: 30 de mayo de 2017 


\section{Introducción}

Los moluscos son de gran importancia para el hombre, por ser un recurso económico y gastronómico explotable. Además, son relevantes en el ecosistema acuático porque son buenos indicadores del estado y funcionamiento de los ecosistemas, debido a que son sensibles a las variaciones del medio ya que dependen de ciertas variables fisicoquímicas para su desarrollo (Strayer, 2008).

En Colombia su uso como fuente de alimento se hace casi exclusivamente en forma artesanal en las zonas costeras. Un grupo relevante entre los moluscos son los bivalvos, entre los que sobresale la ostra de agua dulce (A.rivoli) registrada en el río Opia y endémica de la cuenca del río Magdalena (López, Vásquez, Reinoso, Vejarano \& García, 2009). En el departamento del Tolima esta especie se explota a nivel turístico y alimenticio (López, et al., 2009).

Esta especie pertenece al orden Unionoida, comúnmente conocido como mejillones u ostras de agua dulce, este orden es muy diverso y cuenta con más de 150 géneros distribuidos en todos los continentes salvo en la Antártida (Haas, 1969). Este orden está catalogado a nivel mundial por los malacólogos como "en peligro" dado que tiene un valor extremadamente interesante desde la perspectiva biológica, ya que posee un único ciclo de vida que incorpora cuidado parental y parasitismo larval en peces (López, et al., 2009).

La mayoría de los trabajos realizados sobre A. rivoli son muy antiguos y en la actualidad no se cuenta con trabajos ecológicos y mucho menos sobre el estado de la población. Según Granados (1973), los reportes más antiguos que se tienen son las publicaciones realizadas por Ferussac (1823), dÓrbigny (1851) y Yonge (1978) quienes hacen una descripción morfológica de la especie, hablan sobre la procedencia de las conchas y sobre la evolución de la familia Etheriidae. Granados (1973) realizó averiguaciones de posibles sitios en donde pudo existir o existen individuos de A. rivoli, determinando su distribución geográfica, así como algunos aspectos morfológicos y ecológicos.

Laverde (1982) reporto para Colombia algunos géneros de la familia Unionidae incluyendo a A. rivolli. Arteaga (1993), realizó un estudio en el que hace referencia al ciclo de vida de la ostra hasta su fase parasita (lasidium), denotando también algunos aspectos de su morfología, variables fisicoquímicas del hábitat y condiciones para su cultivo. Graf y Cummings (2006) en su trabajo dan algunos aspectos de distribución y diversidad del orden Unionoidea. Bogan y Hoeh (2000) hablan de las relaciones evolutivas entre los géneros de la familia Etheriidae.

Las valvas de A. rivoli se caracterizan por tener formas variadas de acuerdo a la superficie sobre la cual se han fijado, presentando generalmente asimetría entre las mismas, adquiriendo la valva fija la forma del sustrato al crecer sobre éste. La concha es de color verde oliva con líneas concéntricas bien definidas, cuya cara interna presenta una coloración gris metálico y se aprecia fácilmente en ellas la inserción tanto del musculo aductor como del manto.
En la charnela se puede observar un apéndice o gancho el cual puede ser indicador del tamaño de fijación al sustrato (Arteaga-Sogamoso, 1993). Yonge (1978) considera al gancho como la característica más llamativa de la concha, así como que la forma de la concha depende enteramente del hábitat donde crezca la ostra (Figura 1).

En la morfología interna de A. rivoli en el área central de la masa corporal, es posible distinguir fácilmente la presencia de un solo músculo aductor, el cual se haya fijado a las caras internas de las valvas. La presencia de un solo músculo aductor (monomiarianismo) es característico de la especie (Granados, 1973). La masa corporal se encuentra cubierta (excepto el músculo aductor) por los dos lóbulos del manto, que son libres y se extienden desde la parte dorsal cubriéndola en ambos lados (Arteaga-Sogamoso, 1993). A. rivoli se caracteriza por presentar sexos separados. Como en casi todos los bivalvos de agua dulce $A$. rivoli retiene sus embriones y huevos en un marsupio, el cual ocupa gran parte de la extensión dentro de las dos semi-branquias internas (Arteaga-Sogamoso, 1993).

A. rivoli solo ha sido reportada en el departamento del Tolima en los ríos Opia y Cunday (Bonetto, 1997), juega un papel ecológico importante en el ecosistema acuático, además de ser de gran relevancia por su uso comestible y artesanal. El estado actual de la población es completamente incierto debido a que se han realizado estudios de la especie desde hace más de 20 años. Por lo cual es de suma importancia evaluar el estado actual de la población, así como algunos aspectos ecológicos relevantes en la cuenca del río Opia, con el fin de proponer futuros estudios sobre su reproducción, aspectos tróficos, entre otros, que permitan un mayor conocimiento de la especie y a futuro poder generar planes y programas de conservación y manejo de la especie.

Con el fin de determinar el estado actual de la población, se hace necesario evaluar algunos aspectos ecológicos y ambientales que pueden relacionarse con la especie A. rivoli, por tal motivo se generaron las siguientes preguntas; 1 . En qué condiciones (densidad y distribución) se encuentra la población de A. rivoli en la cuenca del río Opia, 2. Existen diferencias a lo largo del gradiente longitudinal de A. rivoli en la cuenca del río Opia y 3. Esta la distribución de A. rivoli determinada por las variables ambientales presentes en la cuenca del río Opia. Para resolver estas preguntas se evaluó la distribución espacial y vertical a lo largo de un gradiente longitudinal, así como las posibles relaciones entre A. rivoli y las variables fisicoquímicas de la cuenca del río Opia.

\section{Materiales y métodos}

Área de estudio. La cuenca del río Opia se encuentra localizada al noroeste del departamento del Tolima, abarcando los municipios de Ibagué y Piedras. El área de la cuenca es de aproximadamente $325,16 \mathrm{~km}^{2}$, con una longitud aproximada de 65 km, una elevación media de 593,5 m y una pendiente superficial media de $18,60 \%$, caracterizando el terreno como suavemente inclinado. Nace a los 1038 m de altura y desemboca (en el río Magdalena) a los 254 


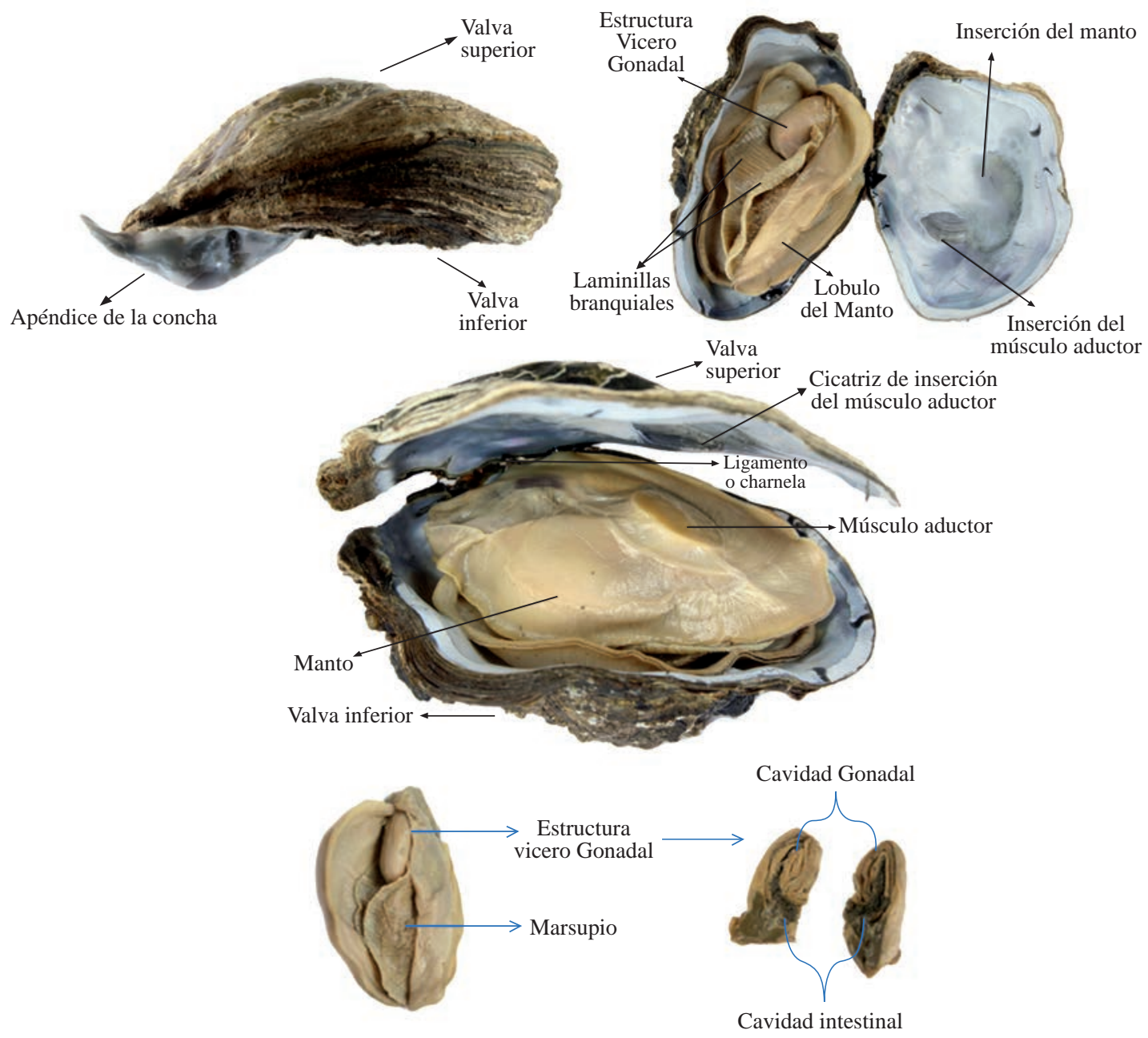

Figura 1. Características morfológicas externas e internas de Acostaea rivoli del río Opia.

m. La cuenca del río Opia se caracteriza por dos tipos de zonas de vida, el bosque húmedo pre-montano, el cual tiene una temperatura promedio de 18 a $24 \mathrm{C}^{\circ}$, con promedios anuales de precipitación entre 1000 y 2000 mm anuales. La selección de las estaciones se llevó a cabo teniendo como base el área del río, la intervención antropogénica, el acceso al río, entre otros. Se determinaron 18 estaciones de muestro a lo largo de la cuenca desde los 230 m (desembocadura al río Magdalena) hasta los 936 m (Figura 2).

Trabajo de campo. Se llevó a cabo un muestreo de 12 días entre los meses de septiembre y octubre de 2009, donde se establecieron 18 estaciones de muestreo en las cuales se evaluó la distribución espacial y el tamaño de la población de A. rivoli. Adicionalmente, se realizó el conteo de los especímenes en 10 transectos longitudinales usando para ello un cuadrante de $1 \mathrm{~m}^{2}$, realizando un conteo visual por unidad de esfuerzo de acuerdo a la metodología propuesta por Smith (2006). Adicionalmente, se evaluaron profundidades de 1 a $3 \mathrm{~m}$, dependiendo de las características del sitio de muestreo, con el fin de determinar la distribución vertical de la especie (Krebs, 1998).
En cada estación de muestreo paralelo a la colecta del material biológico se tomaron muestras de agua (4 litros), para la determinación de alguna variables fisicoquímicas del agua, tales como; temperatura, turbiedad, conductividad, dureza, oxígeno disuelto, porcentaje de saturación de oxígeno, $\mathrm{pH}$, alcalinidad, nitratos, nitrito, fosfatos, demanda química de oxígeno, sólidos totales, sólidos suspendidos y sólidos disueltos. Así mismo, fueron tomadas muestras de agua en frascos esterilizados para la evaluación de coliformes fecales y totales.

Trabajo de laboratorio. Los individuos fueron medidos y pesados para determinar la talla promedio; midiendo el ancho, alto y longitud de los individuos de $A$. rivoli. Se determinó el peso completo (concha y partes blandas), el peso de las partes blandas y el peso del complejo gonadal.

Análisis de datos. Para determinar la distribución espacial y vertical, se realizó un análisis descriptivo de los datos utilizando la densidad de la especie en cada una de las estaciones y profundidades evaluadas. Con el fin de determinar el estado de la población se establecieron tallas promedio y biomasa de los individuos. Para determinar 


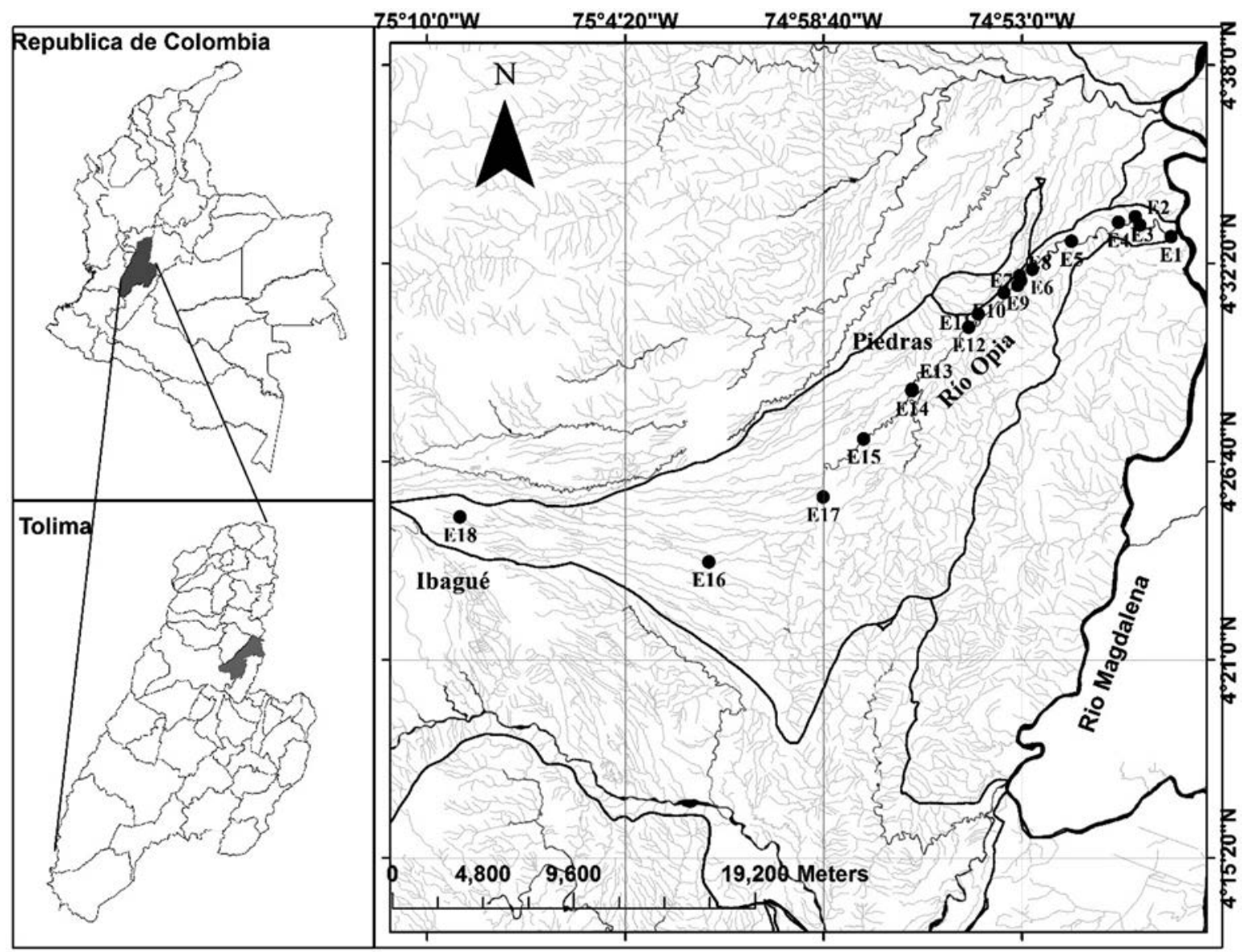

Figura 2. Estaciones de muestreo en la cuenca del río Opia-Tolima

diferencias estadísticas en las densidades de la especies en las diferentes profundidades se utilizó un análisis de varianza no paramétrico de Kruskal-Wallis. Con el fin de identificar las variables con mayor influencia sobre las dinámicas fisicoquímicas de la cuenca se realizó un análisis de componentes principales. Posteriormente, para determinar si existía alguna relación entre la densidad de $A$. rivoli y las variables fisicoquímicas se realizó un análisis de correlación de Spearman (no paramétrico) ya que las variables no presentaron una distribución normal. Las pruebas se realizaron mediante el paquete estadístico $\mathrm{R}$ usando la librería Vegan (R Development Core Team 2016).

\section{Resultados}

Distribución de la Población. Se observaron 303 organismos en 18 estaciones. La mayor densidad se presentó en la estación Lorencito con 6,67 ind $/ \mathrm{m}^{2}$ y la menor en las estaciones Puente Doima, Molino Federal y Quebrada Opia con 0 ind $/ \mathrm{m}^{2}$ (Tabla 1). La mayoría de los individuos se encuentran viviendo por separado y ocasionalmente forman colonias en las que las ostras jóvenes se adhieren a las conchas de las ostras más viejas (Yonge, 1978). A. rivoli se encuentra en profundidades que varían entre 1 y $3 \mathrm{~m}$, generalmente fijos sobre rocas cuyo diámetro varía entre 0,3 y $2 \mathrm{~m}^{2}$. En el perfil vertical la mayor densidad se presentó en el primer metro con 29, $62 \mathrm{ind} / \mathrm{m}^{2} \mathrm{y}$ la menor en el tercer metro con 4,50 ind $/ \mathrm{m}^{2}$ (Figura 3). La prueba de KruskalWallis mostró diferencias significativas en la profundidad entre las estaciones $($ Test statistic $=11,9349$, P-Value $=$ 0,00256082) (Figura 4).

Acostaea rivoli presenta un rango de peso completo de 11,1 a 142,2 gr con un peso promedio de 50,61 gr $(n=64)$. El rango de peso para las partes blandas es de 1,8 a 15,2 gr con un promedio de 8,04 gr $(n=64)$. El rango para el complejo gonadal es de 0,5 a 4,7 gr con un promedio de 1,87 gr $(n=64)$, siendo mayores los pesos en individuos maduros. El rango de talla oscilo entre 5,0 y $11,7 \mathrm{~cm}$ de longitud con un promedio de $8,1 \mathrm{~cm}(\mathrm{n}=64)$, un rango de 3,1 a 7,3 $\mathrm{cm}$ de ancho con un promedio de $5,4 \mathrm{~cm}(\mathrm{n}=64)$, y un rango de 1,5 a $4,8 \mathrm{~cm}$ de altura con un promedio de $2,5 \mathrm{~cm}(\mathrm{n}=64)$.

Aspectos fisicoquímicos y su relación con Acostaea rivoli. El comportamiento de las variables fisicoquímicas de la cuenca del río Opia puede corroborarse con el análisis de componentes principales (ACP), el cual mostró que el factor 1 representa el 43,6\% y el factor 2 el 23,4\%, acumulando así el $67 \%$ de la variación total de los datos. El primer factor se caracterizó por variables como alcalinidad $(0,933)$, dureza $(0,99)$, turbidez $(-0,93)$, nitratos $(-0,99)$ y coliformes $(-0,90)$. 
Tabla 1. Densidad de la población de A. rivoli en la cuenca del río Opia-Tolima.

\begin{tabular}{|c|c|c|c|}
\hline Estación & Densidad $\left(\mathrm{m}^{2}\right)$ & Altura (m) & Coordenadas \\
\hline Bocas de Opia & 0,77 & 230 & $4^{\circ} 33$ ' 05.7'” N 74o 48' 44.1'”W \\
\hline El Caucho & 0,80 & 263 & $4^{\circ} 33^{\prime} 25.4^{\prime \prime} \mathrm{N} 740$ 49' $37.8^{\prime \prime} \mathrm{W}$ \\
\hline Charco Redondo & 1,93 & 267 & $4^{\circ} 33^{\prime} 39.9^{\prime \prime} \mathrm{N} 74^{\circ} 49^{\prime} 45.6^{\prime \prime} \mathrm{W}$ \\
\hline El Guadual & 0,16 & 277 & $4^{\circ} 33$ ' $30.2^{\prime \prime} \mathrm{N} 74^{\circ} 50^{\prime} 14.5^{\prime \prime} \mathrm{W}$ \\
\hline Nemesito & 0,17 & 321 & $4^{\circ} 32^{\prime} 58.0^{\prime \prime} \mathrm{N} 74^{\circ} 51^{\prime} 34.7^{\prime \prime} \mathrm{W}$ \\
\hline El Caracolí & 0,08 & 365 & $4^{\circ} 32^{\prime} 09.7^{\prime \prime} \mathrm{N} 74^{\circ} 52^{\prime} 42.2^{\prime \prime} \mathrm{W}$ \\
\hline La Jabonera & 0,08 & 375 & $4^{\circ} 31^{\prime} 58.6^{\prime \prime} \mathrm{N} 74^{\circ} 53^{\prime} 03.9^{\prime \prime} \mathrm{W}$ \\
\hline Los Tres Pegaos & 2,10 & 380 & $4^{\circ} 32^{\prime} 01.2^{\prime \prime} \mathrm{N} \quad 74^{\circ} 53^{\prime} 16.4^{\prime \prime} \mathrm{W}$ \\
\hline El Remolino & 3,20 & 386 & $4^{\circ} 32^{\prime} 00.3^{\prime \prime} \mathrm{N} 74^{\circ} 53^{\prime} 15.3^{\prime \prime} \mathrm{W}$ \\
\hline Lorencito & 6,67 & 397 & $4^{\circ} 31^{\prime} 38.7^{\prime \prime} \mathrm{N} 74^{\circ} 53^{\prime} 43.7^{\prime \prime} \mathrm{W}$ \\
\hline El Platanal & 3,30 & 428 & $4^{\circ} 31^{\prime} 05.9^{\prime \prime} \mathrm{N}$ 74 54' 22.2” $\mathrm{W}$ \\
\hline Tovar & 3,60 & 432 & $4^{\circ} 30^{\prime} 37.1^{\prime \prime} \mathrm{N} 74^{\circ} 54^{\prime} 43.5^{\prime \prime} \mathrm{W}$ \\
\hline Bocas de Doima & 2,20 & 499 & $4^{\circ} 28^{\prime} 54.5^{\prime \prime} \mathrm{N} 74^{\circ} 56^{\prime} 20.6^{\prime \prime} \mathrm{W}$ \\
\hline Quebrada Doima & 3,20 & 501 & $4^{\circ} 28^{\prime} 55.6^{\prime \prime} \mathrm{N} 74^{\circ} 56^{\prime} 18.6^{\prime \prime} \mathrm{W}$ \\
\hline El Tambor & 0,93 & 541 & $4^{\circ} 27^{\prime} 17.4^{\prime \prime} \mathrm{N} 74^{\circ} 57^{\prime} 22.5^{\prime \prime} \mathrm{W}$ \\
\hline Puente Doima & 0,00 & 626 & $4^{\circ} 25^{\prime} 51.8^{\prime \prime} \mathrm{N} 74^{\circ} 58^{\prime} 51.9^{\prime \prime} \mathrm{W}$ \\
\hline Molino Federal & 0,00 & 689 & $4^{\circ} 23^{\prime} 58.3^{\prime \prime} \mathrm{N} 75^{\circ} 02^{\prime} 08.2^{\prime \prime} \mathrm{W}$ \\
\hline Quebrada Opia & 0,00 & 936 & $4^{\circ} 25^{\prime} 04.6^{\prime \prime} \mathrm{N} 75^{\circ} 09^{\prime} 03.4^{\prime \prime} \mathrm{W}$ \\
\hline
\end{tabular}

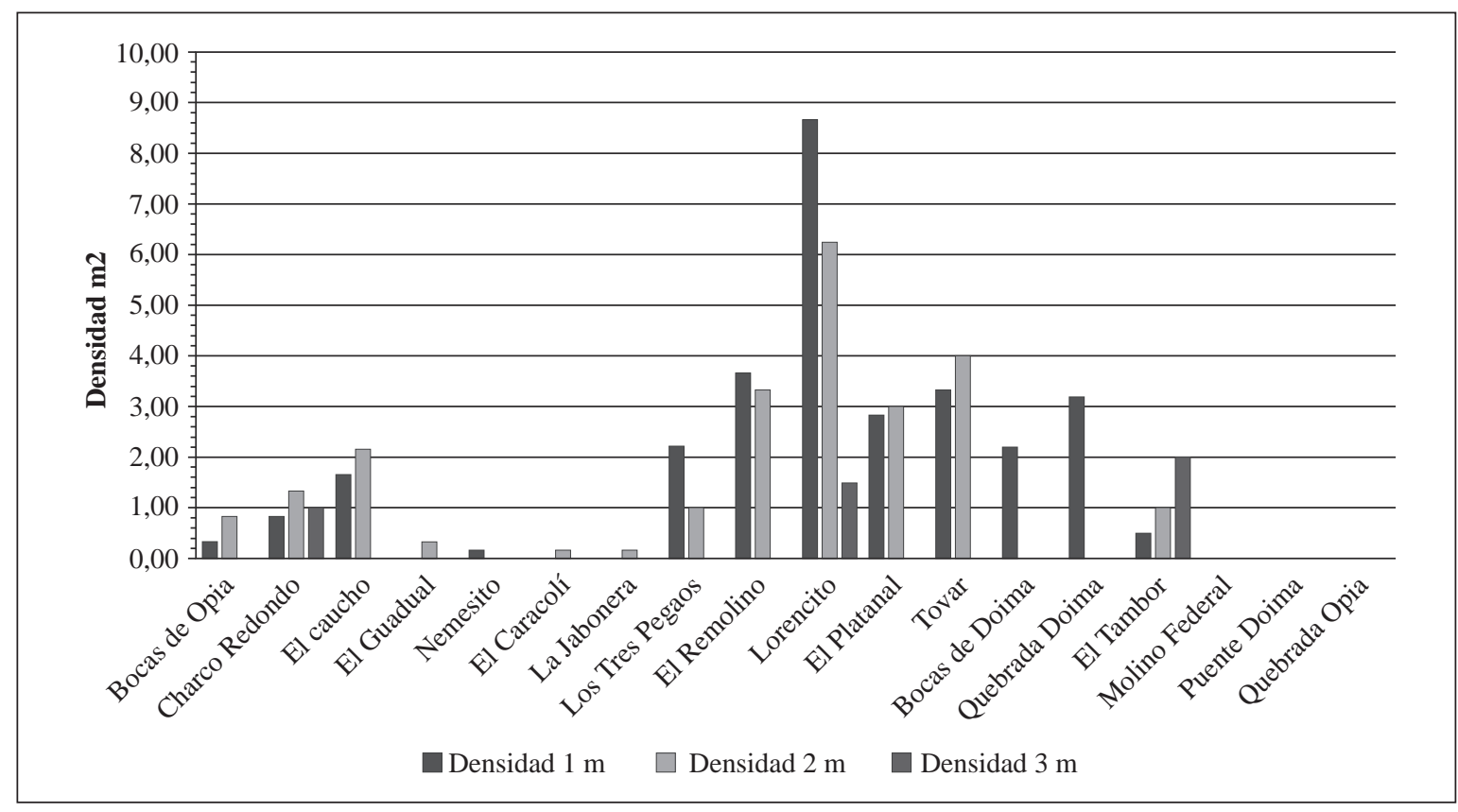

Figura 3. Densidad de la población de A. rivoli en el perfil vertical

El segundo factor solo aporto una variable nueva altura $(-0,85)$. En el diagrama de ordenación se evidenciaron dos grupos, el primero ubicado a la izquierda, caracterizado por las variables coliformes, sólidos totales, turbidez y nitratos, en este grupo se encuentran las estaciones que tienen niveles elevados de estas variables, lo que puede significar gran cantidad de materia orgánica en el cuerpo de agua. El grupo 2 ubicado a la derecha, está relacionado con las variables $\mathrm{pH}$, alcalinidad y dureza, las estaciones que se asocian a estas variables son las que presentan los valores más altos, evidenciando así posibles procesos de mineralización en estas localidades (Figura 5). 


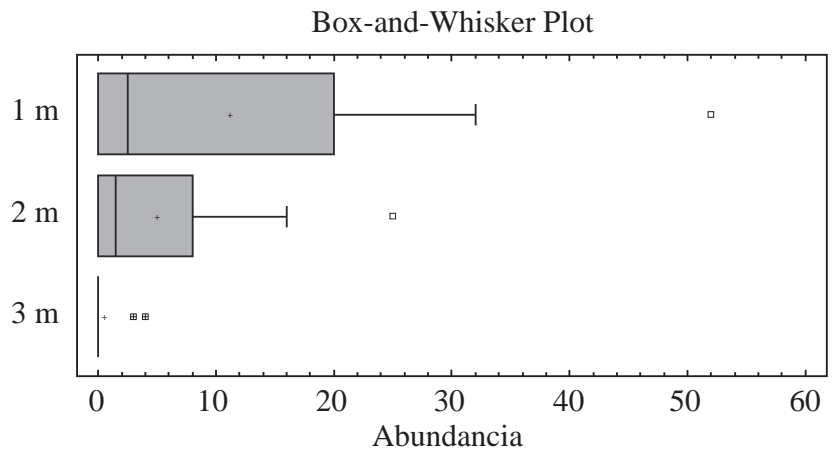

Figura 4. Diagrama de caja y bigotes para la profundidad en las diferentes estaciones evaluadas. Se indica el valor promedio y el límite de confianza del 95\%.

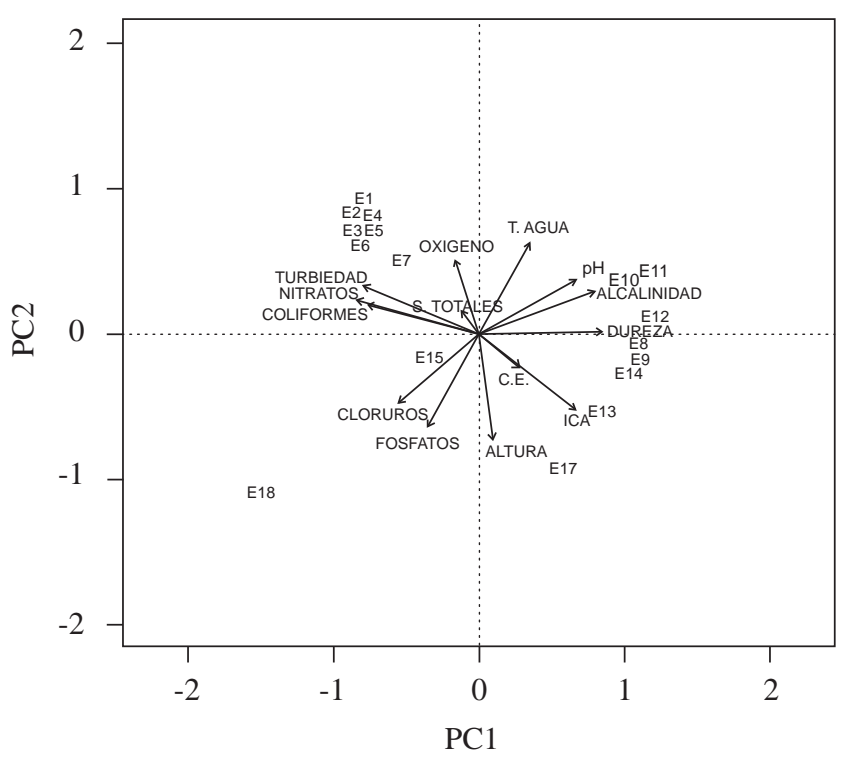

Figura 5. Diagrama de ordenación de las variables fisicoquímicas en los componentes I y II del análisis de componentes principales en la cuenca del río Opia.

Con el fin de determinar si existe alguna relación entre la densidad de A. rivoli y las variables fisicoquímicas se realizó un análisis de correlación de Spearman. Este análisis mostro una correlación significativa de la densidad con algunas variables. Se observó una correlación positiva con el $\mathrm{pH}$, la conductividad, la alcalinidad y la dureza, todas con un coeficiente de correlación superior al 50\%. Las variables cloruros, fosfatos, nitratos y coliformes presentaron una correlación negativa con la densidad y con coeficientes de correlación superiores al 50\% (Tabla 2) (Figura 6).

\section{Discusión}

Con relación a la densidad de ostras por estación, la localidad que registró la mayor densidad fue la estación Lorencito, lo que probablemente está relacionado con sus características fisicoquímicas las cuales podrían ser adecuadas para el óptimo desarrollo de la especie (Strayer, 2008), pues registró los valores más altos de dureza, alcalinidad y $\mathrm{pH}$. Esta estación presentó una zona de bosque que no ha sido muy impactada debido a que es de difícil acceso para el hombre, permitiendo que la población se encuentre en buenas condiciones. La baja densidad en Nemesito, Caracolí y la Jabonera probablemente se debe a que son estaciones cercanas al casco urbano, zonas impactadas por actividades agrícolas, turismo y sobre explotación de la especie. Las estaciones que no presentaron organismos son las de mayor altitud (626 a $936 \mathrm{~m}$ ), las cuales presentan características topográficas distintas ya que son quebradas sin presencia de paredes inclinadas (peñas), por lo cual no hay presencia de un sustrato adecuado para el establecimiento de la especie.

Tabla 2. Correlación de Spearman entre las 18 variables fisicoquímicas y la densidad de A. rivoli en la cuenca del río Opia.

\begin{tabular}{lcc}
\hline Variable & Spearman & p-valor \\
\hline Altura (m) & $-0,124$ & 0,623 \\
Temperatura agua & 0,451 & 0,061 \\
\hline pH & 0,714 & $\mathbf{0 , 0 0 1}$ \\
Conductividad & 0,600 & $\mathbf{0 , 0 0 9}$ \\
Oxígeno disuelto & 0,348 & 0,157 \\
\hline Turbiedad & $-0,414$ & 0,087 \\
Alcalinidad & 0,698 & $\mathbf{0 , 0 0 1}$ \\
\hline Dureza & 0,650 & $\mathbf{0 , 0 0 4}$ \\
Cloruros & $-0,530$ & $\mathbf{0 , 0 2 4}$ \\
Sólidos totales & 0,143 & 0,571 \\
Fosfatos & $-0,603$ & $\mathbf{0 , 0 0 8}$ \\
Nitratos & $-0,490$ & $\mathbf{0 , 0 3 9}$ \\
Coliformes totales & $-0,540$ & $\mathbf{0 , 0 2 1}$ \\
\hline ICA & 0,395 & 0,105 \\
\hline
\end{tabular}

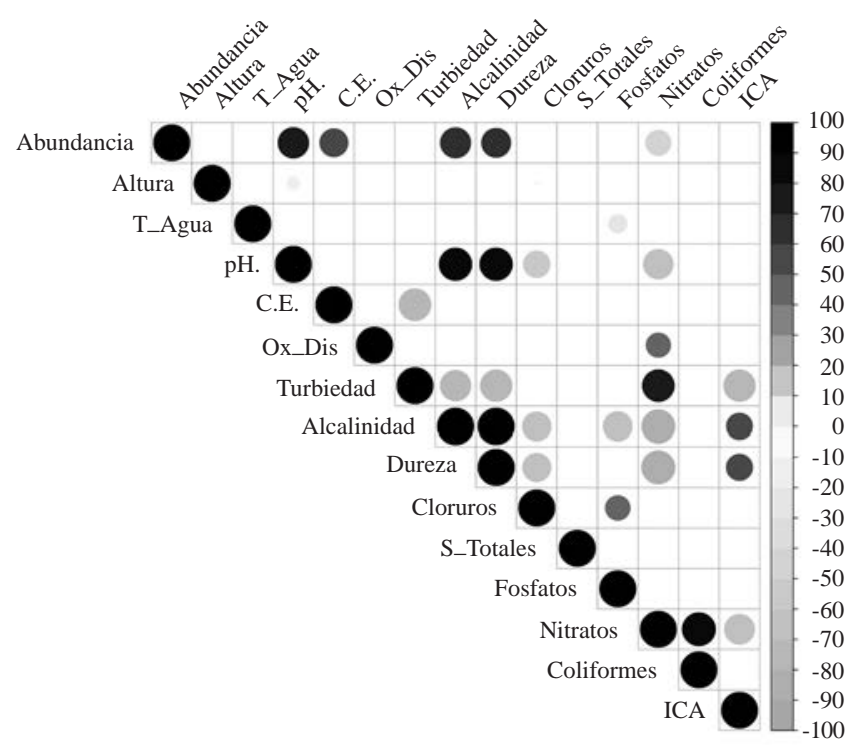

Figura 6. Gráfico de correlación de Spearman. 
La cuenca del río Opia cuenta con una alta densidad de organismos según lo expuesto por Villela y Smith (2005), ya que la presencia de $\geq 1$ ostra $/ \mathrm{m}^{2}$ es signo de alta densidad. El número de animales vivos es mayor en cuevas y en las grietas de las rocas, ya que son zonas de difícil acceso para el hombre (Arteaga-Sogamoso, 1993; López, et al., 2009). Sin embargo la sobreexplotación a la que se ve sometida la especie como fuente de alimento puede llevar a una drástica disminución de su densidad, así como los impactos en el hábitat por extracción de agua para riego, adecuación de balnearios para recreación y a la contaminación de las aguas por aguas residuales y agrícolas (Granados, 1973; Strayer, 2008, Bogan, 2008).

La mayor densidad en el primer y segundo metro se debe probablemente a la oferta de alimento, ya que $A$. rivoli se alimenta de fitoplancton y este se encuentra en mayor número en la zona fótica. Durante el primer metro las comunidades fitoplantónicas son más productivas ya que las condiciones de luz y temperatura son óptimas para su desarrollo (Roldán \& Ramírez, 2008), proporcionando así, condiciones ideales para que la población de A. rivoli se establezca y desarrolle un gran número de individuos.

La gran densidad de organismos en el primer metro concuerda con lo reportado por Granados (1973) y Yonge (1978), quienes ubican a la especie en aguas poco profundas y rara vez a profundidades mayores a un metro. El tamaño de los ejemplares recolectados posiblemente está indicando que la población se encuentra envejecida, esto se debe a que la tasa de crecimiento del orden Unionidae es muy baja, los individuos crecen de 1 a 2 mm por año (Neves \& Moyer, 1988) y al solo encontrarse organismos de tallas superiores a $5 \mathrm{~cm}$ podría indicar que la mayoría de los organismos son adultos, con una edad aproximada de 25 a 50 años.

El análisis de componentes principales muestra que existe una posible descarga de materia orgánica en las aguas del río Opia, provocando un incremento de microorganismos descomponedores que causan desequilibrios en el ecosistema, reflejando un cambio en la calidad del agua (Roldán \& Ramírez, 2008). En el grupo de la izquierda (Figura 5) encontramos las estaciones que presentan una mayor actividad antrópica (Agricultura, descarga de aguas residuales, etc.) lo que ocasiona el incremento en los coliformes y una menor densidad de organismos. La dureza y la alcalinidad denotan procesos de mineralización de las aguas (Ramírez \& Viña, 1998), por lo que en el río Opia, este proceso se presenta en mayor medida debido a su formación geológica (lecho de rocas y cantos rodados), que a su vez libera iones (carbonato de calcio) que aumentan el $\mathrm{pH}$, dureza y alcalinidad del agua.

Las variables con correlación positiva en el análisis de Spearman, intervienen en los procesos de mineralización de la cuenca y denotan la cantidad de calcio disponible en el río, lo que afecta directamente a la especie. Después de la alimentación, los factores más importantes para los unionoidos son el oxígeno para la respiración y el calcio para el crecimiento de la concha. La dureza está directamente relacionada con la cantidad de calcio presente en el agua, por lo que valores mayores a $150 \mathrm{mgCaCO}_{3} \mathrm{~L}^{-1}$ denotan un agua dura.

El río Opia presentó un rango de agua de moderadamente dura a dura (143 a $192 \mathrm{mgCaCO}_{3} \mathrm{~L}^{-1}$ ), por lo que la presencia de calcita $\left(\mathrm{CaCO}_{3}\right)$ está muy relacionada con la formación geológica, de la naturaleza del suelo y del grado de meteorización de las rocas. Todos estos factores ayudan a que en la cuenca el ión calcio sea elevado favoreciendo así la formación de las conchas en A. rivoli (Roldán \& Ramírez, 2008). Los uniónidos son capaces de sobrevivir en concentraciones de calcio $<5 \mathrm{mg} / \mathrm{L}$, sin embargo, concentraciones por debajo de $1 \mathrm{mg} / \mathrm{L}$ (aguas suaves) pueden afectar, disminuir y restringir la distribución de la población; algunas especies del orden se limitan exclusivamente a aguas ricas en calcio (Strayer, 2008). Hunter (1964) plantea que la concentración requerida para la mayoría de los moluscos es $>20 \mathrm{mg} / \mathrm{L}$, por lo que aguas duras pueden sostener mejor las poblaciones de moluscos.

La alcalinidad proporciona la acción buffer que amortigua cambios de $\mathrm{pH}$ en el agua; así, conociendo la alcalinidad de un cuerpo de agua se tiene una idea de la capacidad que tienen estos de mantener los procesos biológicos, además de una productividad constante y duradera (Roldán \& Ramírez, 2008). El pH presentó un rango de 7,2 a 8,7 unidades tendiendo siempre a la alcalinidad. Las variaciones bruscas del $\mathrm{pH}$ afectan la flora y la fauna acuática al variar las concentraciones de carbono inorgánico total, los valores de $\mathrm{pH}$ por fuera de un rango entre 6,5 y 8 disminuyen la diversidad por estrés fisiológico (Roldán \& Ramírez, 2008).

El pH al estar relacionado con la alcalinidad se comporta de forma estable a lo largo de las estaciones por lo que se encuentra en el rango aceptable para su supervivencia. Las características geológicas e hidrológicas de la cuenca permiten el establecimiento de $A$. rivoli, ya que las concentraciones de calcio son altas y permiten el sostenimiento de la especie, además la meteorización que ejerce el agua en las rocas permite que $A$. rivoli tome los iones necesarios para su formación y desarrollo.

Las variables con correlación negativa (fosfatos, nitratos y cloruros) son factores que se relacionan con la cantidad de nutrientes disponibles en el río, ya sea de forma natural o por escorrentía de los cultivos aledaños al este. La asociación de estas variables con la densidad de A. rivoli es inversamente proporcional. El aumento en la carga de materia orgánica y nutrientes puede provocar una disminución en las concentraciones de oxígeno, que puede ser suficiente para afectar a las ostras juveniles impidiendo su desarrollo (McMahon \& Bogan, 2001).

La presencia de la especie en las estaciones parece estar relacionada con la dureza y consistencia del sustrato. Strayer (2008) expone que sustrato adecuado es aquel que proporcione soporte (suficientemente firme para la fijación), que sea estable (que permanece en su lugar durante las inundaciones) y por donde hay flujo de alimentos y materiales 
esenciales (oxígeno, calcio, etc). La cuenca del río Opia al tener formaciones rocosas denominadas peñas en la mayor parte de su extensión, presenta las características adecuadas de soporte y estabilidad para que $A$. rivoli se distribuya adecuadamente a lo largo de la cuenca, esto sumado a que la cuenca posee altas concentración de iones de calcio.

\section{Conclusiones}

Las características geológicas de la cuenca del río Opia permiten que ciertas características físicas y químicas del río, como la dureza, el oxígeno y el sustrato rocoso ayuden al sostenimiento de los individuos de A. rivoli. Debido a la formación rocosa de la cuenca, el agua presenta un porcentaje alto de calcio, una buena oxigenación y proporciona además un sustrato adecuado para el establecimiento de la población. Con relación a la distribución vertical de la especie el primer metro de profundidad fue el más utilizado para el establecimiento, ya que el sustrato es más adecuado para su fijación y hay una mayor disponibilidad de alimento.

En trabajos futuros con $A$. rivoli se recomienda realizar un seguimiento de la densidad de la población en la cuenca, con el fin de identificar si existe algún grado de amenaza sobre la especie debido a la sobreexplotación por parte de los habitantes de la región. Adicionalmente, es importante realizar estudios más profundos con respecto a la historia de vida de A. rivoli para determinar algunos aspectos ecológicos importantes en aras de la conservación de la especie.

\section{Agradecimientos}

Agradecemos el apoyo financiero de la Corporación Autónoma Regional del Tolima, al Comité Central de Investigaciones de la Universidad del Tolima y al Grupo de Investigación en Zoología de la Universidad del Tolima. Expresamos un agradecimiento especial a Iván Gonzales y Néstor Oviedo por su apoyo en campo.

\section{Conflicto de intereses}

No declarado

\section{Referencias}

Arteaga-Sogamoso, E. (1993). Contribución al conocimiento del desarrollo larval de la ostra de agua dulce Acostaearivoli (Deshayes, 1827) (Bivalvia, Etheriidae) en condiciones de laboratorio y algunos aspectos de su biología reproductiva. Fundación Universidad de Bogotá Jorge Tadeo Lozano. Santa fe de Bogotá.

Bogan, A. E. (2008). Global diversity of freshwater mussels (Mollusca, Bivalvia) in freshwater. Hydrobiologia, 595 (1): 139-147.

Bogan, A. E., \& Hoeh, W. R. (2000). On becoming cemented: evolutionary relationships among the genera in the freshwater bivalve family Etheriidae Bivalvia: Unionoida). Geological Society, London, Special Publications, 177 (1): 159-168.

Bonetto, A. A. (1997). The freshwater oysters (Muteloidea: Mutelidae). Their taxonomy and geographic distribution in the whole of the naiads of the world. Biociencias, 5 (1): 113-142.
D'Orbigny, A. (1851). Note sur un nouveau genre de Coquille lamellibranche d'eau douce découvert dans les rivières de la Nouvelle-Grenade par M. le colonel ACOSTA, et décrit par M. Alcide d’ORBiGNY. Revue et Magasin de Zoologie. Pure et Appliquées. ser 2, vol 3: 183-188.

Ferussac, J. B. (1823). Notice sur les Ethéries trouvées dans le Nil par M. Cailliaud. Mémoires de la Société d’Histoire Naturelle 1: 353-369.

Graf, D. L. \& Cummings, K. S. (2006). Palaeoheterodont diversity (Mollusca: Trigonioida+Unionoida): what we know and what we wish we knew about freshwater mussel evolution. Zoological Journal of the Linnean Society, 148: 343-494.

Granados, H. (1973). Distribución hidrográfica y ecología de A. rivoli (Deshayes) de la cuenca del río Magdalena, Colombia (Bivalvia, Etheriidae). Ciencia, 28: 1-16.

Gutiérrez-Hoyos, N. E. (2011). Aspectos bioecológicos de la ostra de agua dulce (Acostaea rivoli) en la cuenca del río Opia, Departamento del Tolima. Universidad del Tolima. Ibagué. Colombia.

Haas. (1969). Superfamilia Unionacea. Das Tierreich (Berlin) Lieferung 88: Seite I-X: 1-663.

Hunter, W.R. (1964). Physiological aspects of ecology in nonmarine molluscs. En K.M. Wilbur \& C.M. Yonge (eds). (1964). Physiology of Mollusca. (pp.83-126). New York and London. Academic Press.

Krebs, C. J. (1998). Ecological methodology (2 ed.).United states of America. Benjamin Cummings. Menlo Park, California.

Laverde, H.A. (1982). Datos sobre colección, inventario y cría artificial de bivalvos de agua dulce en Colombia, Scientidae, 1 (2): 165-172.

López-Delgado, E.O., Vasquez-Ramos, J.M., Reinoso-Florez, G., Vejarano-Delgado, M.A., \& Garcia-Melo, J. E. (2009). Plan de Manejo de la Ostra de agua dulce Acostaea rivoli (Deshayes,1827) del río Opia, Departamento del Tolima. Ibagué-Tolima. León Graficas.

McMahon R. F., \& Bogan, A. E. (2001).Mollusca: Bivalvia. En J. H. Thorp \& A. P. Covich (eds). Ecology and Classification of North American Freshwater Invertebrates (2nd Edition). (pp.331-429) San Diego- California. Academic Press.

Neves, R. J., \& Moyer, S. N. (1988). Evaluation of techniques for age determination of freshwater mussels(Unionidae). American Malacological Bulletin, 6 (2): 179-188.

R Development Core Team. (2011). R: A language and Environment for Statistical Computing. R Foundation for Statistical Computing: Vienna, Austria.

Ramírez, A., \& Viña, G. (1998). Limnología Colombiana: aportes a su conocimiento y estadísticas de análisis. Bogotá: Fundación Universidad de Bogotá Jorge Tadeo Lozano.

Roldán, G., \& Ramírez, J. (2008). Fundamentos de Limnología neotropical. Colombia Segunda Edición. Medellín. Editorial de la Universidad de Antioquia.

Smith, D. R. (2006). Survey design for detecting rare freshwater mussels. Journal of the North American Benthological Society. 5 (3): 701-711.

Strayer, D. L. (2008). Freshwater mussel ecology: a multifactor approach to distribution and abundance (Vol 1). University of California Press, Berkeley, Los Angeles, London.

Villella, R. F., \& Smith, D. R. (2005). Two-phase sampling to estimate river-wide populations of freshwater mussels. Journal North American Benthological. 24 (2): 357-368.

Yonge, M. (1978). On the monomyarian, Acostaea rivoli and evolution in the family Etheriidae (Bivalvia: Unionacea). Journal of Zoology, London, 184 (4): 429-448.

Young, M. R., \& J. C. Williams (1984). The reproductive biology of the freshwater pearl mussel Margaritifera margaritifera (Linn.) in Scotland. I. Field studies. Archiv für Hydrobiologie, 99: $405-422$ 